Studies in African Linguistics

Volume 32, Number 2, 2003

\title{
WHY KISWAHILI ADOPTED THE WORDS FOR SIX, SEVEN AND NINE ${ }^{*}$
}

\author{
Aimee Johansen \\ University of Illinois, Urbana-Champaign
}

\begin{abstract}
This squib addresses the topic of the numeral system of Kiswahili, in which some numerals are of Bantu origin and others are borrowed from Arabic. The main issue of interest is the following: Why did Kiswahili adopt the Arabic words for 'six', 'seven' and 'nine', when Kiswahili words already existed for these numerals? Little has been written on this topic, other than simple descriptions of the facts. I argue that sita 'six' and saba 'seven' were borrowed to replace the existing Bantu forms because they fit better into the pattern of disyllabic words found in the Kiswahili numbers from 1 to 10 . The word for 'eight' was not borrowed because the Bantu word already fit this pattern. I also demonstrate that tisa 'nine' was borrowed into the language later than sita and saba, and probably for different reasons.
\end{abstract}

There is a long history of commercial and cultural contact between East African coastal people and Arabs from the Gulf Region, dating back at least to the first century A.D., seven centuries before the arrival of Islam in East Africa. One result of this long-term contact was a large degree of borrowing of Arabic lexemes into Kiswahili (Bosha 1993), including a significant part of the numeral system, which contains elements of both Bantu and Arabic origin. The breakdown in the

* I would like to thank the following professors and students at the University of Illinois for their assistance: Elabbas Benmamoun, Eyamba Bokamba, Jennifer Cole, Alma Gottlieb, Brent Henderson, Hans Henrich Hock, and Heidi Lorimor. Audiences at the $35^{\text {th }}$ Annual Conference on African Linguistics and the Center for African Studies brown bag series at the University of Illinois also provided valuable input for the paper. Stéphane Alnet and Elizabeth Zwanziger assisted in proofreading and formatting. All mistakes are, of course, my own. 
numeral system of standard spoken Kiswahili from 1 to 10 is as in (1) (Ashton 1944: 50-1).

$\begin{array}{lll}\text { (1) Bantu origin } & \text { Arabic origin } & \\ \text {-moja } & & \text { 'one' } \\ \text {-wili } & & \text { 'two' } \\ \text {-tatu } & & \text { 'three' } \\ \text {-(n)ne } & & \text { 'four' } \\ \text {-tano } & \text { 'five' } \\ & \text { sita } & \text { 'six' } \\ \text {-nane } & \text { saba } & \text { 'seven' } \\ & \text { tisa } & \text { 'eight' } \\ \text { kumi } & & \text { 'nine' } \\ & & \text { 'ten' }\end{array}$

The focus of this paper is the borrowing pattern between 'six' and 'nine'. Bantu numerals are used for 'one' through 'five', for 'eight' and for 'ten'. Numerals borrowed from Arabic are used for 'six', 'seven' and 'nine'. Given the tendency outlined by Greenberg (cited in Denning \& Kemmer 1990: 305) that languages generally borrow numerals in a sequence, this pattern of borrowing is unusual. However, as demonstrated below, tisa 'nine' was borrowed later than sita 'six' and saba 'seven', and for different reasons, which obviates the need for an explanation of why 'six', 'seven' and 'nine' were borrowed, but not 'eight'.

Below, I examine why Kiswahili adopted the Arabic words for 'six', 'seven' and 'nine' when Bantu forms already existed in the language. Numerals are generally borrowed to fill gaps in the lexicon of a language, or else to simplify the numeral system. However, literary Kiswahili has Bantu forms for all of the numerals, so the borrowing that occurred in spoken Kiswahili cannot be explained in terms of gap filling. Some of the Bantu forms remain in the spoken language, and others were replaced by Arabic forms. The table in (2) gives an overview of Bantu and Arabic forms available for 'one' through 'ten' in literary Kiswahili (Knappert 1990:52-3), which is naturally more conservative than spoken Kiswahili, preserving older forms. Preferred spoken forms are underlined. 
Bantu origin

(2) moja, moya, -mwe

pili, mbili, -wili

tatu

ine, nne

$\underline{\operatorname{tano}}$

tundatu

fungate

nane

kenda

kumi
Arabic origin

wahidi, ahidi

thineni, thinani

thalatha, salasa

arubaa

hamsa

$\underline{\text { sita }}$

saba, sabaa

thamani, samanya

tisa, tisaa, tisia

ashara 'one'

'two'

'three'

'four'

'five'

'six'

'seven'

'eight'

'nine'

'ten'

Greenberg remarked that numerals are usually borrowed in a sequence; in fact, he pointed to Kiswahili as being exceptional, as it borrows 'six' and 'seven' from Arabic, then uses a Bantu numeral for 'eight', and again borrows an Arabic numeral for 'nine'.

The Bantu words tundatu 'six' and fungate 'seven' were replaced by words whose Kiswahili adaptations have the form $\mathrm{CVCV}$. In addition to satisfying the well-established universal preference for CV syllables, the borrowed forms, sita and saba, fit in better with the disyllabic pattern of the numerals of Bantu origin (-moja 'one', -(w)ili 'two', -tatu 'three', -(n)ne 'four', -tano 'five', -nane 'eight' and kumi 'ten'), 'than their replaced Bantu counterparts.

Bantu origin word

te.nda.tu

fu.nga.te
Kiswahili adaption of Arabic word

si.ta

sa.ba

The ability of sita and saba to fit in with the overall phonological pattern of the Kiswahili numerals was probably a factor in their replacing their Bantu counterparts, which did not match this pattern.

Since sita 'six' and saba 'seven' were borrowed from Arabic, we must ask why thamani 'eight' was not borrowed as well. The Bantu-origin word for 'eight', -nane, already fit well into the disyllabic pattern described above, so there was no reason to adopt the trisyllabic thamani of Arabic.

\footnotetext{
' I would like to thank an anonymous reviewer for pointing out that Kiswahili numerals fit in nicely with the tendency for counting systems to have a rhythmic or rhyming pattern.
} 
Tisa entered the language later than sita and saba. Kiswahili is the only one of several Guthrie (1967-71) group G Bantu languages examined ${ }^{2}$ that has tisa for 'nine', rather than kenda, shenda or some similar variant. Additionally, Shaba Kiswahili (spoken in the Democratic Republic of Congo), has both kenda and tisa available for 'nine' (Kaji 1985: 363), and Ashton (1944:50) lists kenda as a possibility for 'nine' in her description of Kiswahili. These facts demonstrate that tisa was borrowed more recently than the well-established sita and saba.

Nevertheless, the question remains why tisa was borrowed at all. Knappert (1990) says, "There is no explanation for the fact that Kiswahili speakers preferred e.g. tisa to kenda, but that is a psychological question". In searching for a more satisfactory explanation, we would first expect 'nine' to have some special significance, either religious or cultural, in order for the Arabic tisa to replace the Kiswahili kenda, in spite of the fact that the latter fits nicely into the disyllabic pattern of Kiswahili numerals. However, there is no obvious cultural significance for the number 'nine' in either Swahili or Arab culture. Alternatively, we might expect kenda to resemble some other word that might cause ambiguity or embarrassment if used for 'nine'. This turns out to be more appropriate, since kenda resembles the noun kende 'scrotum, testicle.' It is possible that this played a role in the choice of speakers to use tisa rather than kende, similar to the way that English speakers eliminated all words with a short vowel in the context [f_k], so as to avoid embarrassing associations (Hock \& Joseph 1996: 223).

Phonological and cultural factors appear to account for the nature of numeral borrowing from Arabic to Kiswahili: first, the preference for disyllabic words in Kiswahili numerals; and second, taboo avoidance in the choice of tisa over kenda for 'nine'. More important, the fact that tisa 'nine' was borrowed later than the sequence 6-7 resolves the problem of why 'six', 'seven' and 'nine' were borrowed from Arabic, but not 'eight'.

2 Languages studied include the following Bantu languages classified as group $G$ languages in Guthrie's (1967-1970) widely recognized classification of African languages: Shimaore, Shinzwani, Shingazidja (G40); Kiboondéi and Shambala (G20). 


\section{REFERENCES}

Ashton, E.O. 1944. Swahili Grammar (Including Intonation). London: Longmans, Green and Co., Ltd.

Bosha, Ibrahim. 1993. Taathira za Kiarabu katika Kiswahili Pamoja na Kamusi Thulathiya (Kiswahili-Kiarabu-Kiingereza) [The Influence of Arabic Language on Kiswahili With a Trilingual Dictionary (Swahili-ArabicEnglish)]. Dar es Salaam: Dar es Salaam University Press.

Denning, Keith \& Suzanne Kemmer (eds.). 1990. On Language: Selected Writings of Joseph H. Greenberg. Stanford: Stanford University Press.

Guthrie, Malcolm. 1967-71. Comparative Bantu: An Introduction to the Comparative Linguistics and Prehistory of the Bantu Languages. Farnborough: Gregg International Publishers.

Hock, Hans Henrich \& Brian D. Joseph. 1996. Language History, Language Change, and Language Relationship: An Introduction to Historical and Comparative Linguistics. Berlin: Mouton de Gruyter.

Kaji, Shigeki. 1985. Deux mille phrases de swahili tel qu'il se parle au Zaïre. (African Languages and Ethnography XIX, edited by Morimichi Tomikawa.) Tokyo: Institute for the Study of Languages and Cultures of Asia and Africa (ILCAA).

Knappert, Jan. 1990. "A grammar of literary Swahili". Working Papers in Kiswahili, 10: 1-120.

Department of Linguistics

University of Illinois

Urbana, IL 61801

alnet@uiuc.edu received June 20, 2004

accepted October 19, 2004 
